\section{A preliminary trial to determine whether prevention of dark adaptation affects the course of early diabetic retinopathy}

GB Arden'1, MK Gündüz², A Kurtenbach ${ }^{3}$, M Völker ${ }^{4}$, E Zrenner ${ }^{3}$, SB Gündüz ${ }^{5}$, Ü Kamis², BT Öztürk ${ }^{2}$ and S Okudan²

\begin{abstract}
This study was designed to determine whether a new form of treatment of diabetic retinopathy (DR) was acceptable to patients and whether reduction in the maximal activity of rods in diabetes could affect the progress of DR.

Methods In 12 patients, trans-lid retinal illumination of one eye was employed during sleep to prevent the depolarisation of rods and thus reduce their metabolic activity. Techniques A headband was used to place a source of chemical light over one eye, with its fellow as a control.
\end{abstract}

Measurements Colour contrast thresholds were measured before and after a period of treatment in treated eyes, and the changes were compared to those in untreated fellow eyes, and areas of 'dark retinal anomalies' (microaneurysms, dot haemorrhages) were measured at the same time points.

Results Patients found this intervention to be acceptable, and no adverse effects were noted. In the majority of cases, and for each outcome measure, the treated eyes improved relative to their fellows. The intervention significantly reduced the tritan thresholds in treated eyes relative to their fellows $(P=0.03)$, and the area of dark retinal anomalies decreased in treated eyes and increased in untreated eyes, with a similar probability. Conclusions The study showed that this intervention is safe. Although the study was not powered to study efficiacy, the results are promising and consistent with other reports that indicate the retina in DR is suffering from hypoxia; however, further trials should be undertaken.

Eye (2010) 24, 1149-1155; doi:10.1038/eye.2009.328; published online 5 February 2010
Keywords: diabetic retinopathy;

dark-adaptation; photoreceptor; dark current; colour vision; microaneurysms

\section{Introduction}

One of the leading causes of blindness worldwide is diabetic retinopathy (DR). The earliest phase, seen in animals, is the overproduction of glial fibrillary protein in Müller cells, but soon after retinal capillary endothelial cells and pericytes show immunocytological changes, and increased TUNEL staining (indicative of apoptosis). Capillary leakage and leucostasis develop. In humans, the earliest clinical signs consist of microaneurysms and later, occlusion of small parts of the retinal capillary network. The small blood vessels of the retina are particularly sensitive to damage. ${ }^{1}$ The most commonly accepted cause of DR is oxidative stress, ${ }^{1}$ but it has also been suggested that other conditions contribute, noteably hypoxia, ${ }^{2}$ and such retinal hypoxia, must be greatly augmented during dark adaptation, ${ }^{3-5}$ when the rod dark current becomes maximal. ${ }^{6-9}$ If so, prevention of dark adaptation should slow or prevent the development of DR. This hypothesis can be tested by sleeping in an illuminated environment because this is the only time most of us ever dark adapt. To ensure constant and known retinal illumination we have devised 'light masks', which contain local light sources and used them in a clinical trial.

\section{Materials and methods}

The work was performed in accordance with the Helsinki agreement. Each patient gave informed consent.
${ }^{1}$ Department of Optometry and Visual Science, School of Community and Health Sciences, City University, London, UK

${ }^{2}$ Department of Ophthalmology, Meram Medical Faculty, Selçuk University, Konya, Türkiye

${ }^{3}$ Centre for Ophthalmology, Institute for Ophthalmic Research, University of Tübingen, Tübingen Germany

${ }^{4}$ Centre for Ophthalmology, University Eye Hospital, Tübingen, Germany

${ }^{5}$ Department of Analytical Chemistry, Faculty of Science, Selçuk University, Konya, Türkiye

Correspondence: GB Arden Department of Optometry and Visual Science, School of Community and Health Sciences,

City University, Northampton Square, Islington,

London EC1 V0 HB, UK

Tel: + 440207040 8863;

Fax: + 4402070408355 .

E-mail: g.arden@

city.ac.uk

Received: 8 October 2009 Accepted: 27 November 2009

Published online: 5 February 2010 


\section{Patients}

Six male and 6 female diabetic patients were recruited. Two had type-II diabetes and were aged 65 at the beginning of the trial. The remainder had type-I with an acute onset at ages ranging from 2 to 46 years. The duration of diabetes was 5-33 years, with a mean of 16.3 years. The mean age at the start of the trial was 40.4 years. All patients had a corrected visual acuity of $20 / 20$ or equivalent (two wore spectacles) that did not alter during the trial. All eyes had mild-to-moderate non-proliferative DR. One eye (at random) was selected for illumination. The fellow eyes were used as controls. All subjects were well controlled metabolically. Their systemic treatment was not changed during the trial.

\section{Fundus examinations}

Two-field 50-degree standard colour fundus photography for diabetic screening was performed in Turkey. The camera angle in Germany was 30 degrees. Quantitative measurements were performed on the digitised fundus photographs using the facilities of AutoCAD. The total lesion areas of 'red dots' were measured in a circular area of retina centred near the fovea. The diameter was about six disc diameters. The measurements were made on greatly enlarged images and lesions as small as approximately $22 \mu \mathrm{m}$ in diameter ( $6^{\prime}$ arc) could be measured. This procedure corresponds to methods employed in the measurement of drusen (in ARM $)^{10}$ and various automated systems, which are designed to diagnose early DR. ${ }^{11-13}$ The measurements were made on images obtained before and after the patient wore the light mask. The German patients wore the light masks for 1 year. In Turkey, the duration was 3 months. The grader of the images was masked with respect to which eye was treated.

\section{Colour contrast discrimination}

Colour contrast discrimination was determined with ChromaTest, a computerised method for measuring colour contrast discrimination, ${ }^{14,15}$ using a 4.5 -degree subtense optotype defined by chromatic difference with the background ( $x=0.33, y=0.33$ ), with dynamic masking luminance noise of up to $40 \%$ luminance contrast. The chromaticity varies along a protan or tritan colour confusion line, chosen to be orthogonal. The maximum possible chromaticity difference determined by the monitor phospor emissions is defined as $100 \%$, and the threshold is expressed as a fraction of this displacement. Test-retest studies show that the threshold of an individual with no eye disease can be reproduced to within $1 \%$ (Figure 1a), and did not vary for a normal observer during the course of the trial.

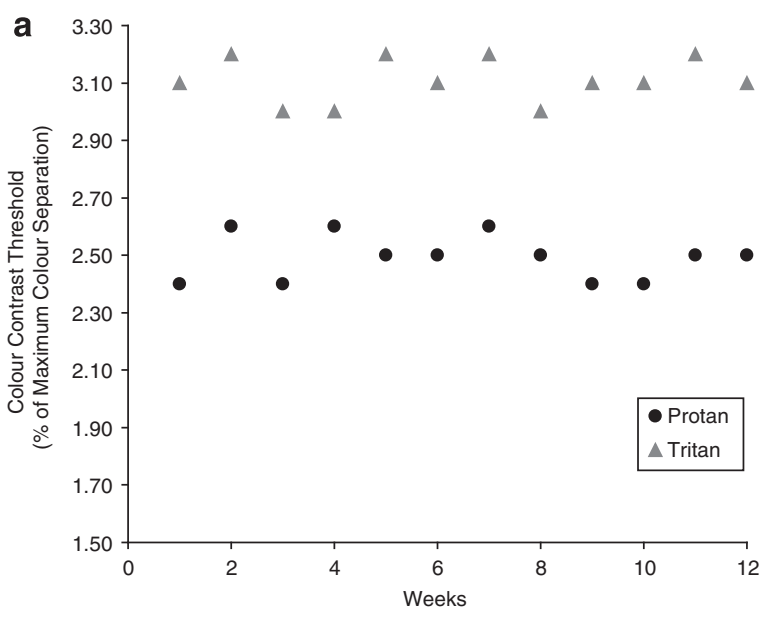

b

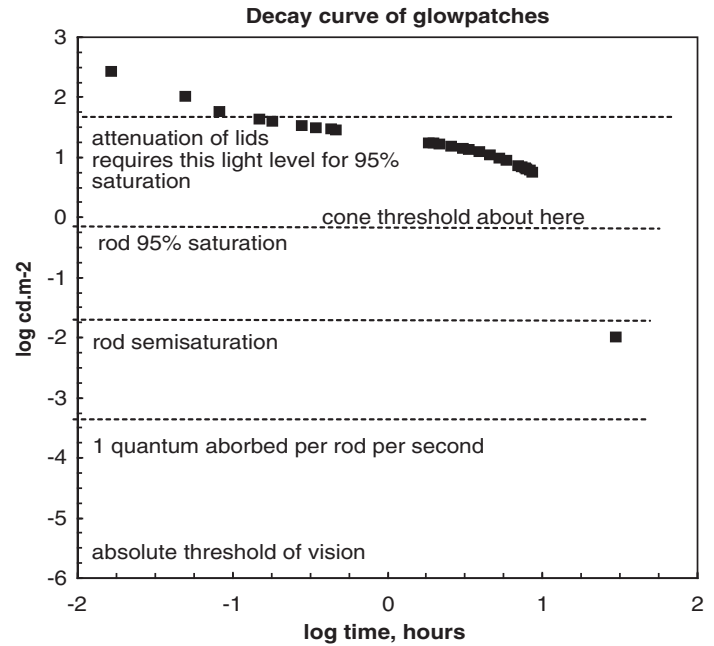

Figure 1 (a) Colour contrast thresholds of normal subject during the course of the trial along the protan and tritan axis. Repeated measurements show no significant change during the course of the trial, and indicate the precision of measurement. (b) The decay curve of the glowpatches used. Note the logarithmic scales. The significance of the horizontal dotted lines is explained in the text.

\section{Light sources}

Light was provided by 'glowpatches'. These safetycertified flat oval sachets, $75 \times 55 \mathrm{~mm}$, are made from inert flexible plastic sheeting containing a dilute solution of phthalates and hydrogen peroxide, together with proprietary catalysts. The reactants (separated by a foil partition, which could be broken by manual compression) mix and emit light of peak output $530 \mathrm{~nm}$. The dominant wavelength was calculated to be $550 \mathrm{~nm}$ after passage of the lids. These sachets were held in place by a light plastic head band that itself was made of inert, medically approved material. Since intense blue and blue-green light is known to have toxic effects on the 
retina, ${ }^{15}$ two of the non-diabetic authors in the study (MK Gündüz and GB Arden), who have no known ocular disease, used these patches in preliminary experiments on one eye during sleep - in the case of MK Gündüz for 3 months (Figure 1a). Figure $1 \mathrm{~b}$ also shows the decay of light output with time in these patches.

\section{Results}

\section{Safety issues}

All patients enrolled in the study used the light sources uninterruptedly and none of them had any ocular or systemic complications during the study. After an initial trial lasting 1 week, the patients found they were able to sleep comfortably while wearing the mask, with a patch over one eye, and there were no drop-outs. None of the patients had any unusual symptoms and all of them were content to be in the trial, in fact some continued after the end of the trial.

\section{Colour vision changes}

The changes in the outcome measures are shown in Table 1 and Figure 2.

Two of the patients (one in each country) had very high tritan thresholds. In one case, this was due to an old haemorrhage, which resolved during the trial, causing a large decrease of threshold. In the other, the patient had extremely high tritan thresholds (approximately 70\%) and although the treated eye improved, we could not exclude some congenital or acquired pathology causing the increase. These two patients were removed from the analysis. Protan thresholds were normal in the eyes of all the remaining patients, and remained unchanged during the trial. Figure 2a shows the mean values and Figure $2 b$ shows the changes with the standard errors. If treated and untreated eyes behaved identically, then the value presented in this figure would be zero, and the variance gives an indication of the uncertainty (noise) of the measurements. It can be seen that intervention caused no significant change.

Tritan thresholds behave in a quite different way. In 9 of 10 patients the difference between the treated and untreated eyes increased, and the figure shows that the change was mostly due to increase in threshold, which did not occur in treated eyes. The magnitude of the change was significantly different to zero by $t$-test. If the results of the two excluded patients were included, 11 of the 12 treated eyes improved relative to their fellows. The magnitude of the average improvement increased, but the variance also increased.

Table 1 Analysis of changes in colour vision

\begin{tabular}{|c|c|c|c|c|c|c|c|c|c|c|c|c|c|c|}
\hline \multirow[b]{3}{*}{ ID } & \multicolumn{7}{|c|}{ Tritan results } & \multicolumn{7}{|c|}{ Protan results } \\
\hline & \multicolumn{2}{|c|}{ Treated eye } & \multicolumn{2}{|c|}{ Untreated eye } & \multirow[b]{2}{*}{$A-B$} & \multirow[b]{2}{*}{$C-D$} & \multirow[b]{2}{*}{$\begin{array}{l}(A-B)- \\
(C-D)\end{array}$} & \multicolumn{2}{|c|}{ Treated eye } & \multicolumn{2}{|c|}{ Untreated eye } & \multirow[b]{2}{*}{$A-B$} & \multirow[b]{2}{*}{$C-D$} & \multirow[b]{2}{*}{$\begin{array}{l}(A-B) \\
(C-D)\end{array}$} \\
\hline & $\begin{array}{c}\text { Start } \\
A\end{array}$ & $\begin{array}{c}\text { End } \\
B\end{array}$ & $\begin{array}{c}\text { Start } \\
C\end{array}$ & $\begin{array}{l}\text { End } \\
D\end{array}$ & & & & $\begin{array}{c}\text { Start } \\
A\end{array}$ & $\begin{array}{c}\text { End } \\
B\end{array}$ & $\begin{array}{c}\text { Start } \\
C\end{array}$ & $\begin{array}{c}\text { End } \\
D\end{array}$ & & & \\
\hline 1 & 10.7 & 8.7 & 8.1 & 11.1 & 2.0 & -3.0 & 5.0 & 3.8 & 3.1 & 5.6 & 5.6 & 0.7 & 0 & 0.7 \\
\hline 2 & 4.9 & 4.4 & 5.8 & 4.0 & 0.5 & 1.8 & -1.3 & 2.7 & 2.9 & 2.9 & 2.2 & -0.2 & 0.7 & -0.9 \\
\hline 3 & 5.9 & 5.5 & 9.9 & 9.6 & 0.4 & 0.3 & 0.1 & 2.3 & 3.6 & 3.1 & 3.1 & -1.3 & 0 & -1.3 \\
\hline 4 & 6.0 & 6.8 & 8.4 & 10.7 & -0.8 & -2.3 & 1.5 & 3.1 & 3.2 & 3 & 3.3 & -0.1 & -0.3 & 0.2 \\
\hline 5 & 5.5 & 7.3 & 5.2 & 7.7 & -1.8 & -2.5 & 0.7 & 3.1 & 3.2 & 3.3 & 3.8 & -0.1 & -0.5 & 0.4 \\
\hline 6 & 8.1 & 7.4 & 25.2 & 28.8 & 0.7 & -3.6 & 4.3 & 2.2 & 3.5 & 2.2 & 3.5 & -1.3 & -1.3 & 0 \\
\hline 7 & 5.7 & 5.5 & 5.5 & 7.1 & 0.2 & -1.6 & 1.8 & 3.8 & 2.2 & 4 & 2.5 & 1.6 & 1.5 & 0.1 \\
\hline 8 & 5.8 & 5.6 & 6.1 & 6.2 & 0.2 & -0.1 & 0.3 & 2.3 & 2.4 & 2.3 & 2.4 & -0.1 & -0.1 & 0 \\
\hline 9 & 8.8 & 8.6 & 8.6 & 8.5 & 0.2 & 0.1 & 0.1 & 3.6 & 3.4 & 3.5 & 3.3 & 0.2 & 0.2 & 0 \\
\hline 10 & 5.5 & 5.6 & 5.6 & 6.2 & -0.1 & -0.6 & 0.5 & 2.1 & 2 & 2 & 2 & 0.1 & 0 & 0.1 \\
\hline Mean & 6.7 & 6.5 & 8.8 & 10.0 & 0.2 & -1.2 & 1.3 & 2.90 & 2.95 & 3.19 & 3.17 & -0.05 & 0.02 & 0.02 \\
\hline SD & 1.9 & 1.4 & 6.0 & 7.0 & 1.0 & 1.7 & 2.0 & 0.67 & 0.56 & 1.05 & 1.04 & 0.85 & 0.73 & 0.73 \\
\hline SE & 0.6 & 0.5 & 1.9 & 2.2 & 0.3 & 0.5 & 0.6 & 0.21 & 0.18 & 0.33 & 0.33 & 0.27 & 0.23 & 0.23 \\
\hline \multicolumn{15}{|c|}{$A-B$ is + ve: The treated eye thresholds decrease over trial } \\
\hline \multicolumn{4}{|c|}{$p$ ( $A$ differs from $B$ ) by chance } & $=$ & \multicolumn{3}{|c|}{0.641} & \multicolumn{4}{|c|}{$p$ ( $A$ differs from $B$ ) by chance } & \multicolumn{2}{|l|}{$=$} & 0.857 \\
\hline \multirow{2}{*}{\multicolumn{4}{|c|}{$\begin{array}{l}p(C \text { differs from } D) \text { by chance } \\
p(((A-B)-(C-D))>0) \text { by chance }\end{array}$}} & $=$ & \multicolumn{3}{|c|}{0.063} & \multicolumn{4}{|c|}{$p$ (C differs from $D$ ) by chance } & \multicolumn{2}{|l|}{$=$} & 0.933 \\
\hline & & & & $=$ & \multicolumn{3}{|c|}{0.03} & \multicolumn{4}{|c|}{$p(((A-B)-(C-D))>0)$ by chance } & $=$ & & 0.359 \\
\hline
\end{tabular}

The bold face indicates the patient who did not improve under the intervention. 


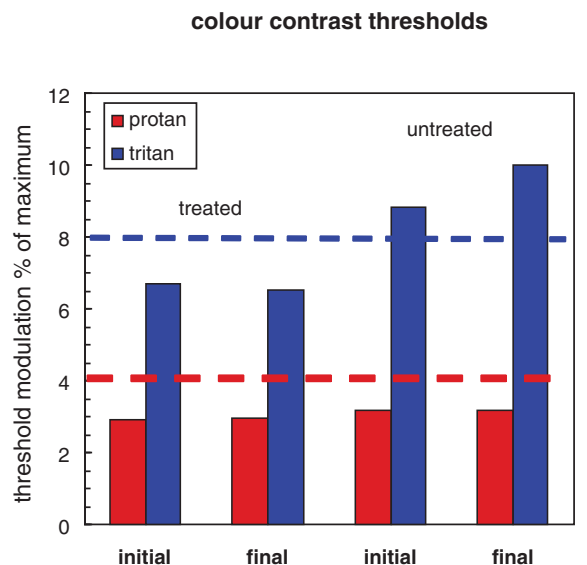

differences between untreated and treated eyes' threshold changes from beginning to end of trial. Positive values indicate success of intervention.

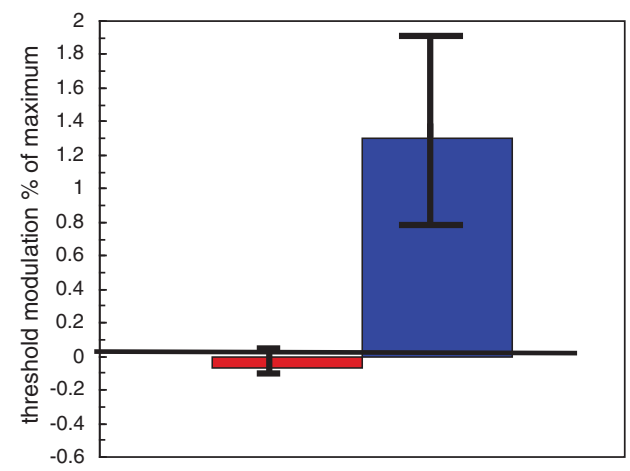

Figure 2 Graphical representation of the data shown in Table 1. Left panel: During the trial protan colour contrast thresholds did not change, but the tritan thresholds rose in untreated eyes. The horizontal lines indicate the upper limits for normal thresholds. Right panel: The difference between before and after-intervention readings for the two eyes. Positive values indicate success of the intervention in preventing rise in colour contrast threshold. The small changes for protan indicate the 'noise' of the measurements. The changes in tritan values are significant.

Table 2 Analysis of changes in area of retinal lesions

\begin{tabular}{|c|c|c|c|c|c|c|c|}
\hline \multirow[b]{3}{*}{ ID } & \multicolumn{7}{|c|}{ Area of dark retinal anomalies - $\%$ of total retinal area } \\
\hline & \multicolumn{3}{|c|}{ Treated eye } & \multicolumn{3}{|c|}{ Untreated eye } & \multirow[b]{2}{*}{$A-B-C+D$} \\
\hline & $\begin{array}{c}A \\
\text { Initial }\end{array}$ & $\begin{array}{c}B \\
\text { Final }\end{array}$ & $\begin{array}{c}A-B \\
\text { Change }\end{array}$ & $\underset{\text { Initial }}{C}$ & $\begin{array}{c}D \\
\text { Final }\end{array}$ & $\begin{array}{c}C-D \\
\text { Change }\end{array}$ & \\
\hline 3 & 0.936 & 0.465 & 0.471 & 0.259 & 0.634 & -0.375 & 0.846 \\
\hline 1 & 0.264 & - & - & 0.497 & 0.731 & - & - \\
\hline 2 & 0.988 & 0.346 & 0.642 & 0.279 & 1.006 & -0.727 & 1.368 \\
\hline 5 & - & 0.329 & - & 0.060 & 0.619 & - & - \\
\hline 11 & 9.691 & 5.203 & 4.488 & 5.203 & 4.825 & 0.379 & 4.109 \\
\hline 5 & 0.549 & 0.326 & 0.224 & 0.295 & 0.740 & -0.445 & 0.668 \\
\hline 6 & 0.744 & 0.316 & 0.428 & 0.277 & 0.265 & 0.012 & 0.416 \\
\hline 6 & 0.214 & 0.068 & 0.146 & 0.414 & 1.037 & -0.623 & 0.769 \\
\hline 12 & 0.395 & 0.445 & -0.050 & 0.240 & 0.475 & -0.235 & 0.185 \\
\hline 8 & 0.176 & 0.171 & 0.005 & 0.051 & 0.022 & 0.029 & -0.024 \\
\hline 7 & 0.190 & 0.232 & -0.042 & 0.611 & 0.654 & -0.043 & 0.001 \\
\hline 10 & 0.307 & 0.154 & 0.153 & 0.059 & 0.022 & 0.037 & 0.116 \\
\hline$n$ & 11 & 11 & 10 & 12 & 12 & 10 & 10 \\
\hline Mean & 1.31 & 0.73 & 0.65 & 0.69 & 0.92 & -0.20 & 0.85 \\
\hline SE & 0.84 & 0.45 & 0.43 & 0.41 & 0.37 & 0.11 & 0.39 \\
\hline \multicolumn{7}{|c|}{ Binomial probability that 9 of 10 treated eyes improve relative to fellows $=$} & 0.01 \\
\hline \multicolumn{7}{|c|}{$t$-test that magnitude of change $(A-B-C+D)>0=$} & 0.03 \\
\hline
\end{tabular}

The bold face indicates the patient who did not improve under the intervention.

\section{Fundus photography}

The fundal appearances of the patients' eyes varied considerably at the beginning of the trial. In only three were there significant exudates, and therefore change in exudates as a function of time were not analysed. The area of microaneurysms and haemorraghes varied between individuals by a factor of $20: 1$, a much greater factor than that observed for colour vision variations (compare Table 1 with Table 2). In only 10 patients were complete records obtained for both eyes. The area of dark anomalies (and the numbers) decreased in the treated eyes over the course of the trial ( $P=0.07$ by $t$-test), while the areas of dark 


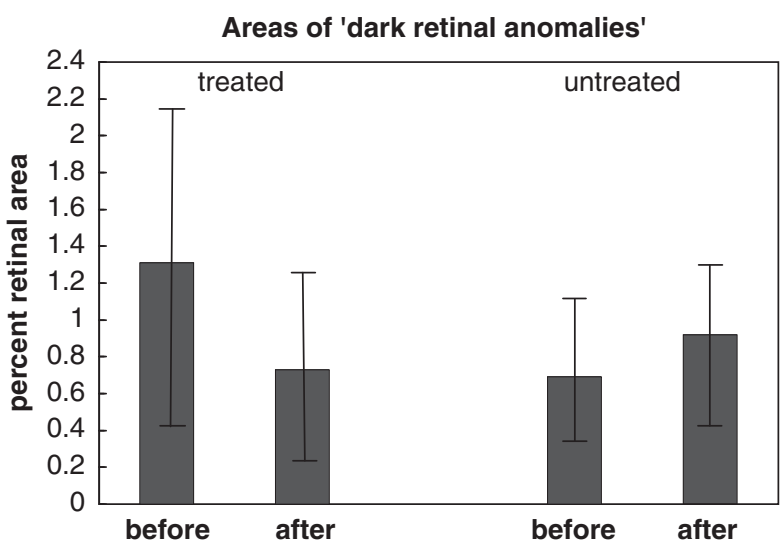

Figure 3 The percentage of retinal area occupied by microaneurysms and dot/blot haemorrhages in the patients eyes before and after treatment.

anomalies increased in untreated eyes (Table 2). In 9 of 10 cases the treated eye appeared to improve more or deteriorate less than the untreated eye. The binomial probability that the intervention was ineffectual and that the asymmetry is due to chance is 0.01 . The probability ( $t$-test) that the difference in column heads $(A-B-C+D)$ is greater than zero is 0.03 . All 10 useable records are included. Figure 3 shows the mean of all values obtained before and after the intervention in all treated and untreated eyes for which data were available.

\section{Discussion}

Although two of the authors conducted preliminary observations on themselves, this is essentially a phase-I trial, and it has shown that patients can be prevented from completely dark adapting for periods of up to 1 year without any apparent adverse effect. Also, it has been shown that this form of treatment is acceptable by patients.

\section{Changes in colour vision}

Phase-I trials do not usually provide evidence about efficacy, but our work indicates that light adaptation reduced the severity of tritan colour vison disturbance in DR. Nine of 10 (or 11 of 12 if the outliers are included). patients' treated eyes improved relative to their untreated fellows. The binomial probability that this could occur by chance is 0.01 . Table 1 suggests that the major effect of treatment was to halt the deterioration found in the untreated eyes. The magnitude of the difference between eyes is also significant by $t$-test (Table 1) The absence of any effect on protan thresholds implies that instrumental changes or biases did not occur. ChromaTest determines the precision with which the threshold determination is made, independently of the colour axis tested, and there are previous reports that in diabetics, blue-yellow deterioration occurs in the absence of red-green changes. ${ }^{16}$ The average thresholds in the patients were comparable to those found earlier in ophthalmic surgeons with diabetes and no visible fundus changes. ${ }^{16}$

\section{Anatomical changes}

A similar trend could be seen in the changes of fundal appearance: the areas of microaneurysms and small dot haemorraghes decreased in the treated eyes and rose in the untreated eyes. The number of such features varied very considerably from patient to patient, over a 20-fold range, as compared with 5-fold for colour vision testing, and thus a phase-II trial powered to assess the difference in outcomes is required to better understand the functional and morphological outcomes. The rate of formation and the persistence of microaneurysms has been analysed and varies considerably with age, type of diabetes, and blood sugar levels. ${ }^{17}$ The increase in time seen in untreated eyes is approximately similar to the findings of Hellstedt and Immonen, ${ }^{17}$ but the decrease in treated eyes is unexpectedly high and one explanation is that in treated eyes no new microaneurysms were formed during the trial and many disappeared. Further analysis was not conducted in view of the small sample size and the considerable difference between patients.

\section{Light levels employed}

This is the first trial that has attempted to measure the quantity of light that could protect the eye in DR. The only available devices produced an illumination that reduced significantly with time as indicated in Figure $1 \mathrm{~b}$. In Figure $1 \mathrm{~b}$ the lowest ordinate level is $1 \mu \mathrm{cd}$, an accepted value for the limit of human vision corresponding to a light level in which one rod in 100 is excited by a quantum every $100 \mathrm{~s}$. Therefore, it is simple to calculate the light intensity that the patch would deliver to the opened eye through a dilated pupil. Despite uncertainties ${ }^{4}$ the initial value achieved by glowpatches is above mesopic levels, and therefore, light adaptation of rods to this level must have occurred for several hours. Such light adaptation would reduce rod dark current although not abolishing it, and this raises the possibility that if higher intensities of light were used, for longer periods, the modest improvement in treated eyes shown by the patients would be more obvious. 


\section{Relationship to previous work and theories of pathogenesis}

The apparent success of the intervention strongly supports the hypothesis that hypoxia during dark adaptation is an important contributory cause of DR. There are a number of reports that point to the same conclusion. Thus, sleeping in the light reduces diabetic macular oedema. ${ }^{18}$ The same author also found that in the small minority of diabetic persons who have suffered from diabetes for $>20$ years, and yet have no retinal abnormality, no less than $62 \%$ sleep in the light. ${ }^{18}$ In addition, oxygen inhalation increases retinal function in diabetics and reduces diabetic macular oedema. ${ }^{19,20}$ The mainstay of surgical treatment, laser pan-retinal photocoagulation, is thought to be successful because it reduces the number of highly active photoreceptors, thus increasing the $\mathrm{pO}_{2}$ by destroying many rods, with improvement in the oxygen supply to the remaining rods. ${ }^{9}$ Conditions under which rod activity is reduced also prevent DR. ${ }^{21-23}$ The loss of hypoxic upregulation of VEGF is found in patients with long standing diabetes and no retinal changes ${ }^{24}$ In diabetic patients who have sleep apnoea, with more than five 'dips' (episodes of reduction of oxygen saturation below $90 \%$ ) per night, ${ }^{25,26}$ the incidence and severity of DR is higher than that in control diabetic subjects. One common factor explains all these observations: the high metabolic rate of rods in darkness places a strain on the normal retina, which in diabetics is unsustainable and results in increased retinal hypoxia, stimulating the production of cytokines, especially vascular endothelial growth factor (VEGF), that contribute to DR.

\section{Importance of the trial}

$\mathrm{DR}$ is a serious complication of a very common disorder, and the prevalence is growing fast. Although treatment is available, it is difficult and expensive to organise screening programmes for the population at risk, and the treatment is invasive and destructive. This trial has shown that with a high degree of probability a different form of treatment is efficacious. Obviously it would be desirable to conduct larger trials, but the fact that significant results have been found with so few patients and with such a short trial, supports the hypothesis that dark adaptation plays an important role in the devleopment of DR. It would be desirable to investigate different patient populations (for example those with diabetic macular oedema) using other measures of outcome such as central macular thickness using optical coherence tomography (OCT). The present work has raised the possibility that inexpensive, simple, prophylactive measures could be devised, which would greatly reduce the incidence of DR.

\section{Summary}

What was known before

- Increase in rod oxygen demand during dark adaptation.

- Circumstantial evidence for retinal hypoxia in diabetic retinopathy (DR).

- Pan-retinal photocoagulation (PRP) is probably effective through relief of retinal hypoxia.

- Anecdotal evidence that increasing hypoxia accelerated DR (and vice versa).

What this study adds

- Patients can sleep in light without adverse effects.

- Dim light apparently reverses or slows the progress of DR in a small, short trial; outcomes tested: alteration of colour vision and area of 'dark vascular anomalies'.

\section{Conflict of interest}

The authors declare no conflict of interest.

\section{Acknowledgements}

This work was supported in part by a grant from Selçuk University Scientific Research Projects Funds (project no. 05401095) and has previously been communicated in part to the Selçuk University/Konya and to Yüksek Öğretim Kurumu/Ankara. Some results have been presented at the 47th symposium of ISCEV, 2009. We acknowledge the gift of modified glowpatches by Omniglow Corporation, which made the project possible. We thank Dr I White, Department of Mathematics, Imperial College London, for statistical advice and Miss S Sivaprasad for critical reading of the paper.

\section{References}

1 Brownlee M. The pathobiology of diabetic complications: a unifying mechanism. Diabetes 2005; 54: 1615-1625.

2 Curtis TM, Gardiner TA, Stitt AW. Microvascular lesions of diabetic retinopathy: clues towards understanding pathogenesis? Eye 2009; 23: 1496-1508.

3 Arden GB, Wolf JE, Tsang Y. Does dark adaptation exacerbate diabetic retinopathy? Evidence and a linking hypothesis. Vision Res 1998; 38: 1723-1729.

4 Arden GB, Wolf JE, Collier J, Wolff C, Rosenberg M. Dark adaptation is impaired in diabetics before photopic loss can be seen. Can hypoxia contribute to diabetic retinopathy?. In: Hollyfield, JG (ed). Retinal Degenerative Disease and Experimental Therapy. Plenum Press: NY, 1999; pp 305-325.

5 Arden GB, Sidman RL, Arap W, Schlingemann RO. Spare the rod and spoil the eye. Br J Ophthalmol 2005; 89: 764-769.

6 Linsenmeier RA. Effects of light and darkness on oxygen distribution and consumption in the cat retina. J Gen Physiol 1986; 88: 521-542.

7 Linsenmeier RA, Braun RD, McRipley MA, Padnick LB, Ahmed J, Hatchell DL, McLeod DS, Lutty GA. Retinal hypoxia in long-term diabetic cats. Invest Ophthalmol Vis Sci 1998; 39: 1647-1657. 
8 Haugh LM, Linsenmeier RA, Goldstick TK. Mathematical models of the spatial distribution of retinal oxygen tension and consumption in the inner and outer retina of the cat including changes on illumination. Ann Biomed Eng 1990; 18 19-36.

9 Yu DY, Cringle SJ. Oxygen distribution and consumption within the retina in vascularised and avascular retinas and in animal models of retinal disease. Prog Retin Eye Res 2001; 20: 175-208.

10 Bird AC, Bressler NM, Bressler SB, Chisholm IH, Coscas G, Davis MD et al. An international classification and grading system for age-related maculopathy and age-related macular degeneration. The International ARM Epidemiological Study Group. Surv Ophthalmol 1995; 39: 367-374.

11 Feman SS, Leonard-Martin TC, Andrews S, Armbruster CC, Burdge TL, Debelak JD, Lanier A, Fischer AG. A quantitative system to evaluate diabetic retinopathy from fundus photographs. Invest Ophthalmol Vis Sci 1995; 36: 174-181.

12 Larsen N, Godt J, Grunkin M, Lund-Andersen H, Larsen M. Automated detection of diabetic retinopathy in a fundus photographic screening population. Invest Ophthalmol Vis Sci 2003; 44: 767-771.

13 Niemeijer M, van Ginneken B, Russell SR, Suttorp-Schulten MS, Abràmoff MD. Automated detection and differentiation of drusen, exudates, and cotton-wool spots in digital color fundus photographs for diagnosis of diabetic retinopathy. Invest Ophthalmol Vis Sci 2007; 48: 2260-2267.

14 Gündüz K, Arden GB, Perry S. A new test of colour vision using TV and computer graphics: results in some common acquired eye diseases. In: Kulikowski JJ, Dickinson CM, Murray IJ (eds). Seeing Contour and Colour. Pergamon Press: Pergamon, NY, 1987, pp 373-380.

15 Gündüz K, Arden GB. Changes in colour contrast sensitivity associated with operating argon lasers. Br J Ophthalmol 1989; 73: $241-246$.

16 Arden GB, Hall MJ. 'Does occupational exposure to argon laser radiation decrease colour contrast sensitivity in UK ophthalmologists?'. Eye 1995; 9: 686-696.
17 Hellstedt T, Immonen I. Disappearance and formation rates of microaneurysms in early diabetic retinopathy. Brit $J$ Ophthalmol 1996; 80: 135-139.

18 Gaynon M. Should people with prethreshold ROP, BDR or ARMD sleep with a nightlight? Review of factors contributing to retinal hypoxia in retinal and choroidal vascular disease. Arvo Abs 2007; E-5122.

19 Nguyen QD, Shah SM, Van Anden E, Sung JU, Vitale S, Campochiaro PA. Supplemental oxygen improves diabetic macular oedema: a pilot study. Invest Ophthalmol Vis Sci 2004; 45: 617-624.

20 Dean FM, Arden GB, Dornhorst A. Partial reversal of protan and tritan colour defects with inhaled oxygen in insulin dependent diabetic subjects. Br J Ophthalmol 1997; 81: $27-30$

21 Arden GB. The absence of diabetic retinopathy in patients with retinitis pigmentosa: implications for pathophysiology and possible treatment. Br J Ophthalmol 2001; 85: 366-370.

22 Stefansson E. Ocular oxygenation and the treatment of diabetic retinopathy. Surv Ophthalmol 2006; 51: 364-380.

23 de Gooyer TE, Stevenson KA, Humphries P, Simpson DA, Gardiner TA, Stitt AW. Retinopathy is reduced during experimental diabetes in a mouse model of outer retinal degeneration. Invest Ophthalmol Vis Sci 2006; 47: 5561-5568.

24 Marsh S, Nakhoul FM, Skorecki K, Rubin A, Miller BP, Leibu R, Levy NS, Levy AP. Hypoxic induction of vascular endothelial growth factor is markedly decreased in diabetic individuals who do not develop retinopathy. Diabetes Care 2000; 23: 1375-1380.

25 Shiba T, Sato Y, Takahashi M. Relationship between diabetic retinopathy and sleep-disordered breathing. Am J Ophthalmol 2009; 147: 1017-1021.

26 Wong A, Merritt S, Butt AN, Williams A, Swaminathan R. Effects of hypoxia on circulating levels of retina-specific messenger RNA in type 2 diabetes mellitus. Ann NY Acad Sci 2008; 1137: 243-252. 